\title{
Variaciones del Canal Mandibular con Importancia Clínica. Reporte de Caso
}

\author{
Anatomical Variations of Mandibular Canal with Clinical Significance. Case Report \\ Ignacio Roa***,**** \& Oscar Arriagada*
}

ROA, I. \& ARRIAGADA, O. Variaciones del canal mandibular con importancia clínica. Reporte de caso. Int. J. Morphol., 33(3):971974, 2015.

RESUMEN: El canal mandibular y su contenido tienen un papel importante en el campo de la cirugía maxilofacial. Este puede presentar variaciones anatómicas, las cuales son clínicamente relevantes principalmente en los procedimientos de implantología. Su desconocimiento ha sido relacionado con complicaciones en la realización de procedimientos quirúrgicos y de anestesia en la práctica dental y maxilofacial. A razón de esto se presenta el reporte de caso de canal mental accesorio y bucle anterior del nervio alveolar inferior.

PALABRAS CLAVE: Canal mandibular; Canal mental; Variación anatómica; Nervio mandibular; Bucle anterior.

\section{INTRODUCCIÓN}

El canal mandibular y su contenido recorren la mandíbula en sentido longitudinal, originandose en el foramen mandibular, para luego descender por el tejido esponjoso del hueso en sentido anteroinferior, siguiendo una curva de concavidad anterosuperior (Rouvière \& Delmas, 1999; Figún \& Garino, 2001; Latarjet \& Ruiz-Liard, 2004). Este puede ser dividido en tres segmentos, siendo el más anterior el que comienza a nivel del segundo premolar donde se bifurca
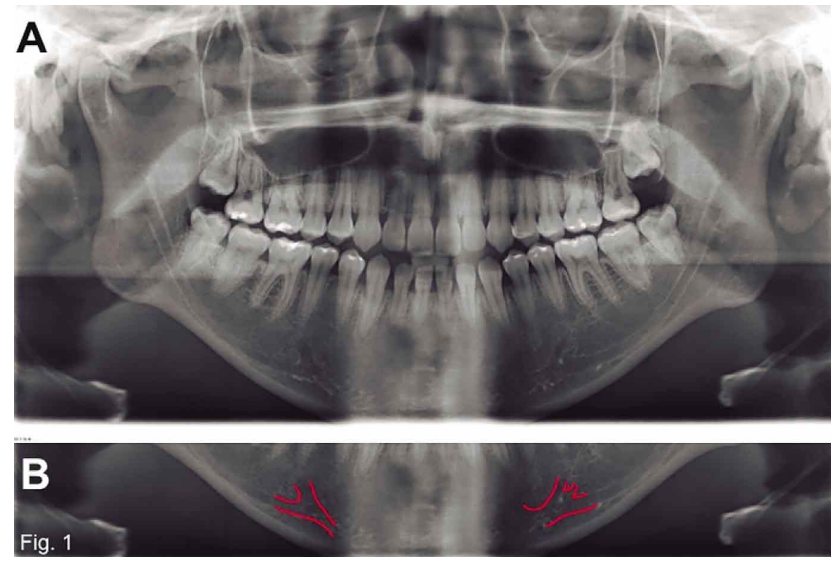

Fig. 1. A. Radiografía panorámica, que muestra bucle anterior bien definido del canal mental de manera bilateral, así como el canal mental accesorio (izquierda). B. Detalle de las variaciones descritas. originando el canal mental y el canal incisivo (Williams et al., 1998; Reiser et al., 2004).

Las variaciones del canal así como de su contenido; nervio mandibular y sus ramas, y los vasos mandibulares han sido reportadas como la posible causa de una inadecuada técnica anestésica; iatrogénia y complicaciones asociadas (Anil et al., 2003; Beltrán et al., 2011), además de complicaciones tales como compresión neurovascular causando entumecimiento y/o dolor. Razón que nos ha llevado a describir el presente caso clínico de un canal mental accesorio, acompañado de bucle anterior del nervio mental de ubicación bilateral.

\section{CASO CLÍNICO}

Paciente de 20 años de edad, en julio de 2014 llega a la consulta privada (Talca, Chile) en busca de atención dental. A través del examen clínico y de una radiografía panorámica digital, se reveló la existencia de un canal mental accesorio a nivel del diente 34 (Fig. 1A y B), además de un bucle anterior del nervio mental bilateral. Clínicamente, en el examen intraoral se observaron caries. Examen periodontal y evaluación de ATM, sin signos o síntomas patológicos.

* Departamento de Ciencias Básicas Biomédicas, Facultad de Ciencias de la Salud, Universidad de Talca, Chile.

** Programa de Doctorado en Ciencias Morfológicas, Facultad de Medicina, Universidad de La Frontera, Temuco, Chile.

**** Becario Conicyt. CONICYT, PFCHA/Doctorado Nacional/2015-21150235. 


\section{DISCUSIÓN}

El conocimiento, tanto de la morfología mandibular, como de las variaciones que ésta puede sufrir, es indispensable para una correcta práctica odontológica.

El canal mandibular se dispone en forma bilateral en la mandíbula; comienza en la cara interna mandibular, se dirige hacia anterior y medial hasta terminar en el foramen mental y contiene tanto el nervio como la arteria y venas alveolares inferiores. Por su ubicación y relaciones, diferentes procedimientos odontológicos, como cirugía de terceros molares, endodoncia e implantes óseo integrados pueden alterar su integridad y dañar tanto los elementos nerviosos como vasculares además de una respuesta anestésica insatisfactoria en técnicas al nervio alveolar inferior (Lew \& Townsen, 2006).

Si bien el canal mental (o mentoniano) es descrito por autores como Testut \& Latarjet (1975), hoy no es contemplado en la Terminologia Anatomica, a pesar de ser una estructura anatómica que describe un trayecto claro y posee un contenido nervioso (Nervus mentalis A14.2.01.094) y vascular (rama de la arteria alveolar inferior A12.2.05.059) (FCAT, 2001).

El estudio detallado tanto de su disposición normal así como también de sus variaciones, puede determinar el éxito o fracaso de un tratamiento, por lo cual hoy en día, la radiografía panorámica o la tomografía computarizada, son utilizadas en la práctica odontológica, proporcionando visibilidad adecuada de las estructuras anatómicas (Jacobs et al., 2004).

El canal y foramen mental deben ser interpretados como el segmento terminal del canal mandibular, siendo descrito el canal como un tubo recurrente o de retorno, ya que por lo general viene del canal mandibular anterior al foramen mental y luego pasa posteriormente hasta alcanzar el foramen mental (Cryer, 1916). Descripción similar a la realizada por otros autores (Warwick, 1950; Yosue \& Brooks, 1989; Mish \& Crawford, 1990).

La presencia de un canal accesorio puede estar acompañado de un foramen mental accesorio; el cual es descrito como aquel foramen que se sitúa generalmente inferior o proximal al foramen mental (Garay \& Cantín, 2014), por el cual también transcurren fibras nerviosas del nervio mental. Olivier (1927) establece que estos se formarían por la separación de un septum óseo en el foramen original, por la persistencia de un agujero anterior o por ser un conducto mandibular de la dentición primaria. Otros autores, confir- man su existencia mediante un examen radiológico, histológico y la disección anatómica mandibular (Sutton, 1974; Farman et al., 1977). Los datos sobre su frecuencia son disímiles, desde el 6,62\% (Shankland, 1994) a no encontrarse (Grover \& Lorton, 1983).

Del punto de vista embriológico se ha propuesto que las variaciones en el canal mandibular se deben a la existencia de tres nervios alveolares inferiores durante el desarrollo prenatal. Estos estarían inervando tres grupos dentarios mandibulares: uno hacia los incisivos un segundo grupo hacia los molares temporales y el último a los molares permanentes (Chavez-Lomeli et al., 1996). Durante la osificación del cartílago mandibular se produciría un lecho óseo alrededor de estas fibras nerviosas fusionadas, constituyendo el canal mandibular. Entonces la existencia de una incompleta fusión de estos nervios alveolares inferiores podría explicar este tipo de variaciones como la descrita en este trabajo (Wadhwani et al., 2008).

En el caso además de presentar un canal mental accesorio, se observaba un bucle o loop anterior del nervio mental, el cual se forma cuando el paquete neurovascular mental cruza inferior y anterior al foramen mental y luego dobla para salir del foramen mental (Bavitz \& Mish, 1993). Este bucle tambien ha sido descrito por otros autores por medio de imaginología (Kuzmanovic et al., 2003; Iyengar et al., 2013) y corroborado por estudios en piezas cadavéricas (Solar et al., 1994) o in vivo (Rosenquist, 1996). Este bucle no es observado clínicamente, aunque puede ser visualizado en aproximadamente 11 a $60 \%$ de las radiografías panorámicas (Ngeow et al., 2009). Por su parte Iyengar et al., describen la presencia de un bucle anterior unilateral en el $21 \%$ de las radiografías panorámicas observadas.

Las alteraciones neurosensoriales en el mentón y el labio inferior son una de las complicaciones más difíciles, pero que en general pasan inadvertidas pudiendo ocurrir durante cualquier procedimiento quirúrgico, como la colocación de implantes, importante en la región premolar inferior. Esta complicación puede ocurrir si las estructuras vitales importantes, como foramen mental y bucle anterior no son identificados y protegidos adecuadamente (Juodzbalys et al., 2010; Singh \& Srivastav, 2010). En consecuencia, para evitar daño a estas estructuras vitales, la localización exacta de las estructuras anatómicas debe ser identificada antes de la cirugía utilizando técnicas imagenológicas apropiadas.

El canal mandibular y sus componentes neurovasculares asociados pueden presentar múltiples variaciones; influenciadas por el sexo, la edad (Suazo Galdames et al., 2007), la raza, la evaluación técnica utilizada y el 
grado de atrofia del hueso alveolar en pacientes desdentados, lo cual puede influir en gran medida en la presentación de estas variaciones (Juodzbalys et al.). Sugerimos que los clínicos debieran identificar cuidadosamente estos puntos anatómicos, por medio del análisis de todos los factores que influyen, previos a los procedimientos de implantología.

ROA, I. \& ARRIAGADA, O. Anatomical variations of mandibular canal with clinical significance. Case report. Int. J. Morphol., 33(3):971-974, 2015.

SUMMARY: The mandibular canal and its contents have an important role in the field of maxillofacial surgery. This can present anatomical variations, which are clinically relevant mainly in implant procedures. Lack of knowledge regarding these variations has been linked to complications in carrying out surgical procedures and anesthesia in dental and maxillofacial practice. Therefore, we report this case of accessory mental canal and mental anterior loop.

KEY WORDS: Mandibular canal; Mental canal; Anatomical variation; Mandibular nerve; Anterior loop.

\section{REFERENCIAS BIBLIOGRÁFICAS}

Anil, A.; Peker, T.; Turgut, H. B.; Gülekon, I. N. \& Liman, F. Variations in the anatomy of the inferior alveolar nerve. Br. J. Oral Maxillofac. Surg., 41(4):236-9, 2003.

Bavitz, J. B.; Harn, S. D.; Hansen, C. A. \& Lang, M. An anatomical study of mental neurovascular bundle-implant relationships. Int. J. Oral Maxillofac. Implants, 8(5):563-7, 1993.

Beltrán, V.; Cantín, M.; Fuentes Fernández, R. \& Engelke, W. Bilateral presence of mandibular incisive canal. An anatomical structure with clinical relevance. Int. J. Morphol., 29(2):5439, 2011.

Chávez-Lomeli, M. E.; Mansilla Lory, J.; Pompa, J. A. \& Kjaer, I. The human mandibular canal arises from three separate canals innervating different tooth groups. J. Dent. Res., 75(8):15404, 1996.

Comité Federal de Terminología Anatómica (FCAT). Terminología Anatómica. Terminología Anatómica Internacional. $1^{\mathrm{a}} \mathrm{ed}$. Madrid, Editorial Médica Panamericana, 2001.

Cryer, M. N. The internal anatomy of the face. 2nd ed. Philadelphia, Lea \& Febiger, 1916.

Farman, A. G.; Nortjé, C. J. \& Grotepass, F. W. Pathological conditions of the mandible: their effect on the radiographic appearance of the inferior dental (mandibular) canal. $\mathrm{Br}$. $J$. Oral Surg., 15(1):64-9, 1977.
Figún, M. \& Garino, R. Anatomía Odontológica Funcional y Aplicada. 2a ed. Buenos Aires, El Ateneo, 2001.

Garay, I. \& Cantín, M. Accessory Mental Foramina Assessed by Cone-Beam Computed Tomography: Report of Unilateral and Bilateral Detection. Int. J. Morphol., 31(3):1104-8, 2013.

Grover, P. S. \& Lorton, L. Bifid mandibular nerve as a possible cause of inadequate anesthesia in the mandible. J. Oral Maxillofac. Surg., 41(3):177-9, 1983.

Iyengar, A. R.; Patil, S.; Nagesh, K. S.; Mehkri, S. \& Manchanda, A. Detection of anterior loop and other patterns of entry of mental nerve into the mental foramen: A radiographic study in panoramic images. J. Dent. Implant., 3(1):21-5, 2013.

Jacobs, R.; Mraiwa, N.; Van Steenberghe, D.; Sanderink, G. \& Quirynen, M. Appearance of the mandibular incisive canal on panoramic radiographs. Surg. Radiol. Anat., 26(4):329-33, 2004.

Juodzbalys, G.; Wang, H. L. \& Sabalys, G. Anatomy of Mandibular Vital Structures. Part II: Mandibular Incisive Canal, Mental Foramen and Associated Neurovascular Bundles in Relation with Dental Implantology. J. Oral Maxillofac. Res., 1(1):e3, 2010 .

Kuzmanovic, D. V.; Payne, A. G.; Kieser, J. A. \& Dias, G. J. Anterior loop of the mental nerve: a morphological and radiographic study. Clin. Oral Implants Res., 14(4):464-71, 2003.

Latarjet, M. \& Ruiz-Liard, A. Anatomía Humana. 2a ed. Buenos Aires, Médica Panamericana, 2004.

Lew, K. \& Townsen, G. Failure to obtain adequate anaesthesia associated with a bifid mandibular canal: a case report. Aust. Dent. J., 51(1):86-90, 2006.

Misch, C. E. \& Crawford, E. A. Predictable mandibular nerve location--a clinical zone of safety. Dent. Today, 9(9):32-5, 1990.

Ngeow, W. C.; Dionysius, D. D.; Ishak, H. \& Nambiar, P. A radiographic study on the visualization of the anterior loop in dentate subjects of different age groups. J. Oral Sci., 51(2):2317, 2009.

Olivier, E. Le canal dentaire inférieur et son nerf chez l'adulte. Ann. Anat. Pathol. (Paris), 4:975-87, 1927.

Reiser, G. M.; Manwaring, J. D. \& Damoulis, P. D. Clinical significance of the structural integrity of the superior aspect of the mandibular canal. J. Periodontol., 75(2):322-6, 2004.

Rosenquist, B. Is there an anterior loop of the inferior alveolar nerve? Int. J. Periodontics Restorative Dent., 16(1):40-5, 1996.

Rouvière, H. \& Delmas, A. Anatomía Humana Descriptiva, Topográfica y Funcional. 10a ed. Barcelona, Masson, 1999. V. 3. 
Shankland, W. E. 2nd. The position of the mental foramen in Asian Indians. J. Oral Implantol., 20(2):118-23, 1994.

Singh, R. \& Srivastav, A. K. Study of position, shape, size and incidence of mental foramen and accessory mental foramen in Indian adult human skulls. Int. J. Morphol., 28(4):1141-6, 2010.

Solar, P.; Ulm, C.; Frey, G. \& Matejka, M. A classification of the intraosseous paths of the mental nerve. Int. J. Oral Maxillofac. Implant.,9(3):339-44, 1994.

Suazo Galdames, I. C.; Morales Herrera, C. A.; Cantín López, M. G. \& Zavando Matamala, D. A. Biometric aspects of the mandibular canal. Int. J. Morphol., 25(4):811-6, 2007.

Sutton, R. N. The practical significance of mandibular accessory foramina. Aust. Dent. J., 19(3):167-73, 1974.

Testut, L. \& Latarjet, A. Tratado de Anatomía Humana. Barcelona, Salvat Editores, 1975. V.1.

Wadhwani, P.; Mathur, R. M.; Kohli, M. \& Sahu, R. Mandibular canal variant: a case report. J. Oral Pathol. Med., 37(2):1224, 2008.

Warwick, $\mathrm{R}$. The relation of the direction of the mental foramen to the growth of the human mandible. J. Anat., 84(2):116-20, 1950 .

Williams, P.; Bannister, L.; Martin, B. \& Gray, H. Anatomía de Gray: Bases anatómicas de la medicina y la cirugía. 38a ed. Madrid, Harcourt Brace, 1998.

Yosue, T. \& Brooks, S. L. The appearance of mental foramina on panoramic radiographs. I. Evaluation of patients. Oral Surg. Oral Med. Oral Pathol., 68(3):360-4, 1989.

\section{Dirección para Correspondencia: \\ Dr. Ignacio Roa Henríquez \\ Unidad de Morfología}

Departamento de Ciencias Básicas Biomédicas

Facultad de Ciencias de la Salud

Universidad de Talca

Av. Lircay s/n, Talca

CHILE

Email. iroa@utalca.cl

Recibido : 05-03-2015

Aceptado: 28-05-2015 